\title{
Irregularity Strength of Corona Product of a Graph with Star Graph
}

\author{
Santhosh Kumar K R \\ Correspondence: Santhosh Kumar K R, Department of Mathematics, University College, Thiruvananthapuram, India. \\ E-mail: santhoshkumargwc@gmail.com
}

Received: September 5, 2015 Accepted: September 21, 2015 Online Published: November 3, 2015

doi:10.5539/jmr.v7n4p97

URL: http://dx.doi.org/10.5539/jmr.v7n4p97

\begin{abstract}
If positive weights are assigned to the edges of a graph $G$, then degree of a vertex is the sum of the weights of edges that are incident to the vertex. A graph with weighted edges is said to be irregular if the degrees of the vertices are distinct. The irregularity strength of a graph is the smallest $s$ such that the edges can be weighted with $\{1,2,3, \cdots, s\}$ and be irregular. This notion was defined by Chartrand et al. (G Chartrand, M. S. Jacobson, J. Lehel, O. R. Ollerman, S. Ruiz \& Saba. 1988). In this paper, we discuss the irregularity strength of corona product of a graph with star graph $K_{1, n}$. We obtain a sufficient condition on the minimum degree of a graph $H$ which determines the irregularity strength of a graph $H$ having $p$ vertices with the star graph $K_{1, n}$.
\end{abstract}

Keywords: Irregularity strength, irregular weighting, Corona product of graphs.

\section{Introduction}

Let $G=(V, E)$ be a graph with at most one isolated vertex and without $K_{2}$ components. A function $f: E \rightarrow Z^{+}$ is called a weighting of $G$, and for an edge $e \in E, f(e)$ is called weight of $e$. The strength $s(f)$ of $f$ is defined as $s(f)=\max _{e \in E} f(e)$. The weighted degree of a vertex $x \in V$ is the sum of weights of its incident edges: $d_{f}(x)=$ $\sum_{e \ni x} f(e)$. We will call it degree of $x$ and is denoted by $w(x)$. The irregularity strength $s(G)$ of $G$ is defined as $s(G)=$ $\min \{s(f), f \quad$ is an irregular weighting of $\mathrm{G}\}$. The study of $s(G)$ was initiated by Chartrand et al. (G. Chartrand, M. S. Jacobson, J. Lehel, O. R. Ollerman, S. Ruiz \& Saba. 1988) and have proved finding irregularity strength is difficult in general. There are not many graphs for which the irregularity strength is known. For an overview of the subject the reader is referred to the paper by G Chartrand et al. (G. Chartrand, M. S. Jacobson, J. Lehel, O. R. Ollerman, S. Ruiz and Saba. 1988).

G. Chartrand et al. (G. Chartrand, M. S. Jacobson, J. Lehel, O. R. Ollerman, S. Ruiz \& Saba. 1988) have proved following propositions

Proposition 1.1 (G. Chartrand, M. S. Jacobson, J. Lehel, O. R. Ollerman, S. Ruiz \& Saba. 1988) Let $G$ be connected graph of order at least 3 containing $p_{i}$ vertices of degree $i$, for some positive integer $i$, then $s(G) \geq \frac{p_{i}-1}{i}+1$

Proposition 1.2 (G. Chartrand, M. S. Jacobson, J. Lehel, O. R. Ollerman, S. Ruiz \& Saba. 1988) For each positive integer $n \geq 2$, there exists a complete network $G$ of order $n$ and strength 2 with degree set $\{n, n+1, n+2, \cdots, 2 n-2\}$ and containing two vertices of degree $\left\lfloor\frac{3 n-2}{2}\right\rfloor$.

Proposition 1.3 (G. Chartrand, M. S. Jacobson, J. Lehel, O. R. Ollerman, S. Ruiz \& Saba. 1988) For each $n \geq 3$, $s\left(K_{n}\right)=3$.

M Jacobson and Lehel (M Jacobson \& J Lehel. 1998) obtained following proposition.

Proposition 1.4 (M Jacobson \& J Lehel. 1998) If $G$ is a graph with $p$ vertices, then $s(G) \geq\lceil\lambda(G)\rceil$, where $\lambda(G)=$ $\max _{i \leq j}\left\{\frac{\left(\sum_{k=i}^{j} n_{k}\right)+i-1}{j}\right\}$, where $n_{k}$ is the number of vertices of degree $k$ in $G$.

R J Faudree et al (R J Faudree, M S Jacobson, L Kinch \& J Lehel. 1991) have proved following proposition.

Proposition 1.5 (R J Faudree, M S Jacobson, L Kinch \& J Lehel. 1991) The irregularity strength of $t K_{3}$ is given by

$s\left(t K_{3}\right)= \begin{cases}\left\lceil\frac{3 t+1}{2}\right\rceil+2 & \text { if } \quad t \equiv 3(\bmod 4) \\ \left\lceil\frac{3 t+1}{2}\right\rceil+1 & \text { otherwise }\end{cases}$

Theorem 1.6 (M I Jinnah \& Santhosh Kumar K R, 2012) If $G=H \odot K_{2}$, where $H$ is a graph with $p \geq 3$ vertices such that $\delta(H) \geq 2$, then $s(G)=p+1$. 
Definition 1.7 The corona $G \odot H$ of $G$ and $H$ is the graph obtained by taking one copy of $G$ (which has $p_{1}$ vertices) and $p_{1}$ copies of $H$, and then joining the $i^{\text {th }}$ vertex of $G$ to every vertex of $i^{\text {th }}$ copy of $H$.

\section{Main Results}

In this section, we consider the class of graphs $G=H \odot K_{1, n}$, where $H$ is an arbitrary graph with $p$ vertices. $G$ has $(n+2) p$ vertices and has $n p$ vertices of degree 2 . By proposition 1.1, $s(G) \geq\left\lceil\frac{n p+1}{2}\right\rceil$. For $n=1, G=H \odot K_{1,1} \cong H \odot K_{2}$. By theorem 1.6, $s\left(H \odot K_{2}\right)=p+1$, if $\delta(H) \geq 2$.

2.1 Cases when $p=1$ and $p=2$

In this section, we consider $p=1$ and $p=2$.

Theorem $2.1 s\left(K_{1} \odot K_{1, n}\right)=\left\lceil\frac{n+1}{2}\right\rceil$ if $n>1$

Proof. $G=K_{1} \odot K_{1, n}$ has $n+2$ vertices and has $n$ vertices of degree 2 . Thus $s(G) \geq\left\lceil\frac{n+1}{2}\right\rceil$.

Let $x_{1}, x_{2}, x_{3}, \cdots, x_{n}$ be the pendant vertices of star, $u$ be the center of star and $v$ be the vertex of $K_{1}$. Edges are $u x_{i}, v x_{i}$ for $i=1,2,3, \cdots, n$ and $u v$.

Define $f: E(G) \rightarrow Z^{+}$by

$$
f\left(u x_{i}\right)=\left\lceil\frac{i}{2}\right\rceil, f\left(v x_{i}\right)=\left\lceil\frac{i+1}{2}\right\rceil \text { and } \quad f(u v)=\left\lceil\frac{n+1}{2}\right\rceil
$$

$w\left(x_{i}\right)=\left\lceil\frac{i}{2}\right\rceil+\left\lceil\frac{i+1}{2}\right\rceil=i+1$, the weights vary of $x_{i}$ vary as $2,3, \cdots, n+1$.

$$
\begin{aligned}
w(u) & =\left(\sum_{i=1}^{n}\left\lceil\frac{i}{2}\right\rceil\right)+\left\lceil\frac{n+1}{2}\right\rceil \\
& =\left\{\begin{array}{lll}
\frac{1}{4}\left(n^{2}+4 n+4\right) & \text { if } & \text { nis even } \\
\frac{1}{4}\left(n^{2}+4 n+3\right) & \text { if } & \text { nisodd }
\end{array}\right. \\
w(v) & =\left(\sum_{i=1}^{n}\left\lceil\frac{i+1}{2}\right\rceil\right)+\left\lceil\frac{n+1}{2}\right\rceil \\
& =\left\{\begin{array}{lll}
\frac{1}{4}\left(n^{2}+6 n+4\right) & \text { if } & \text { nis even } \\
\frac{1}{4}\left(n^{2}+6 n+1\right) & \text { if } & \text { nisodd }
\end{array}\right.
\end{aligned}
$$

As $n>1$, all these weights are distinct. Thus $s(G) \leq\left\lceil\frac{n+1}{2}\right\rceil$. Therefore $s(G)=\left\lceil\frac{n+1}{2}\right\rceil$

Remark 2.2 If $n=1, G=K_{1} \odot K_{2} \cong K_{3}$, then $s(G)=3$.

Now suppose $p=2$, then $H$ is either $K_{2}$ or $\overline{K_{2}}$.

Theorem $2.3 s\left(\overline{K_{2}} \odot K_{1, n}\right)=n+1$ if $n>2$.

Proof. $G=\overline{K_{2}} \odot K_{1, n}$ has $2 n+4$ vertices and has $2 n$ vertices of degree 2 . Then $s(G) \geq n+1$.

Let $x_{i}, y_{i}$ for $i=1,2, \cdots, n$ be the pendant vertices of stars, $x, y$ be the centers of stars and $u, v$ be the vertices of $H$. Edges are $x x_{i}, y y_{i}, u x_{i}, v y_{i}$ for $i=1,2, \cdots, n, x u$ and $y v$.

Define $f: E(G) \rightarrow Z^{+}$by

For $n$ even

For $n$ odd

$$
\begin{aligned}
& f\left(x x_{i}\right)=i, f\left(u x_{i}\right)=i+1, \quad \text { for } \quad i=1,2, \cdots, n \\
& f\left(y y_{i}\right)=i \text { if } i \text { is odd } \\
& f\left(v y_{i}\right)=i+1 \quad \text { if i is even } \\
& =i+1 \text { if } i \text { is even } \\
& f\left(y y_{i}\right)=i \text { for } i=1,3, \cdots, n-2 \\
& =i+1 \text { for } i=2,4, \cdots, n-3 \\
& f\left(y y_{n-1}\right)=n-1 \text { and } f\left(y y_{n}\right)=n+1 \\
& f\left(v y_{i}\right)=i+2 \text { for } i=1,3, \cdots, n-2 \\
& f\left(v y_{n-1}\right)=f\left(v y_{n}\right)=n+1 \\
& f(x u) \quad=f(y v)=n+1
\end{aligned}
$$

Using this assignment, the weights of vertices are calculated as follows.

$w\left(x_{i}\right)=f\left(x_{i} x\right)+f\left(x_{i} u\right)=i+i+1=2 i+1$. These weights vary as $3,5, \cdots, 2 n+1$.

If $n$ is even,

$$
w\left(y_{i}\right)=f\left(y y_{i}\right)+f\left(v y_{i}\right)=2 i+2
$$


If $n$ is odd, $w\left(y_{i}\right)=2 i+2$ for $i=1,2, \cdots, n-2, w\left(y_{n-1}\right)=2 n$ and $w\left(y_{n}\right)=2 n+2$. Thus weights of $y_{i}$ vary as $4,6, \cdots, 2 n+2$.

Also $w(x)=\sum_{i=1}^{n} f\left(x x_{i}\right)+f(x u)=\frac{1}{2}\left(n^{2}+3 n+2\right)$ and

$w(u)=\sum_{i=1}^{n} f\left(u x_{i}\right)+f(x u)=\frac{1}{2}\left(n^{2}+5 n+2\right)$

If $n$ is even $w(y)=\sum_{i=1}^{n} f\left(y y_{i}\right)+f(y v)=\sum_{i o d d} i+\sum_{i \text { even }}(i+1)+n+1=\frac{1}{2}\left(n^{2}+4 n+2\right)$ and $w(v)=\sum_{i=1}^{n} f\left(v y_{i}\right)+f(y v)=$ $\sum_{i \text { odd }}(i+2)+\sum_{i \text { even }}(i+1)+n+1=\frac{1}{2}\left(n^{2}+6 n+2\right)$

If $n$ is odd, $w(y)=\sum_{i=1}^{n} f\left(y y_{i}\right)+f(y v)=\sum_{i \text { odd upto } n-2} i+\sum_{i \text { even upto } n-3}(i+1)+n+1+n-1+n+1=\frac{1}{2}\left(n^{2}+4 n+1\right)$ and $w(v)=\sum_{i=1}^{n} f\left(v y_{i}\right)+f(y v)=\sum_{\text {iodd upto } n-2}(i+2)+\sum_{i \text { even upto } n-3}(i+1)+n+1+n+1+n+1=\frac{1}{2}\left(n^{2}+6 n+3\right)$

Hence if $n$ is even, the weights of vertices are $3,4,5,6, \cdots, 2 n+1,2 n+2, \frac{n^{2}+3 n+2}{2}, \frac{n^{2}+4 n+2}{2}, \frac{n^{2}+5 n+2}{2}, \frac{n^{2}+6 n+2}{2}$. As $n>2$, $2 n+2<\frac{n^{2}+3 n+2}{2}$, so that all weights are distinct. If $n$ is odd, the weights of vertices are $3,4,5,6, \cdots, 2 n+1,2 n+2, \frac{n^{2}+3 n+2}{2}$, $\frac{n^{2}+4 n+1}{2}, \frac{n^{2}+5 n+2}{2}, \frac{n^{2}+6 n+3}{2}$. As $n>2,2 n+2<\frac{n^{2}+3 n+2}{2}$, all these weights are distinct.

Thus $f$ is an irregular weighting of $G$, so that $s(G) \leq n+1$. Hence $s(G)=n+1$ if $n>2$.

Remark 2.4 When $n=1, G=\overline{K_{2}} \odot K_{2} \cong 2 K_{3}$. Then $s(G)=5$ by proposition 1.5 , When $n=2, H=\overline{K_{2}}, G=\overline{K_{2}} \odot K_{1,2}$. $\Delta=3, \delta=2$. If $s=3,3 \Delta-\delta+1=8=$ Number of vertices. So all weights from 2 to 9 are to be included in degree set. $G$ is the disjoint union of two copies of $K_{4}-e$. The weights 2 and 3 will be in one copy of $K_{4}-e$ and the weights 8 and 9 will be in the second copy. Then weights 4 and 5 will be in the second copy. Hence weights 6 and 7 should be in the first copy, which is not possible. Thus $s(G)>3$.

The figure 1 gives an irregular weighting of $G$ so that $s(G)=4$
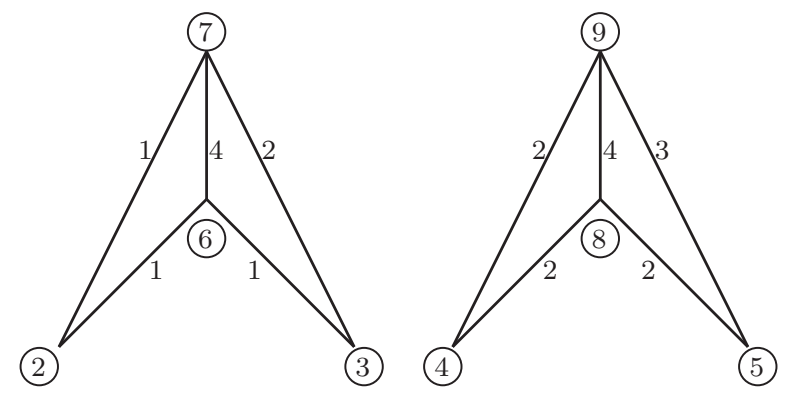

Figure 1. An Irregular assignment of $G=\overline{K_{2}} \odot K_{1,2}$

Theorem $2.5 s\left(K_{2} \odot K_{1, n}\right)=n+1$ if $n>2$

Proof. $G=K_{2} \odot K_{1, n}$ contains one more edge than that of $\overline{K_{2}} \odot K_{1, n}$, the edge of $K_{2}$. Assign the edges of $\overline{K_{2}} \odot K_{1, n}$ as same as in theorem 2.3. Assign the edge of $K_{2}$ with $n+1$. The weights of $x_{i}, y_{i}, x, y$ will be same as that in theorem 2.3.

Also $w(u)=\frac{n^{2}+5 n+2}{2}+n+1=\frac{n^{2}+7 n+4}{2}$ and

$$
w(v)=\left\{\begin{array}{lll}
\frac{n^{2}+8 n+4}{2} & \text { if } & n \text { even } \\
\frac{n^{2}+8 n+5}{2} & \text { if } & n \text { odd }
\end{array}\right.
$$

As $n>2$, all these weights are distinct. Thus $s(G)=n+1$ for $n>2$.

Remark 2.6 For $n=1, G=K_{2} \odot K_{2}$. An irregular weighting of $G$ is given in figure 2 . 


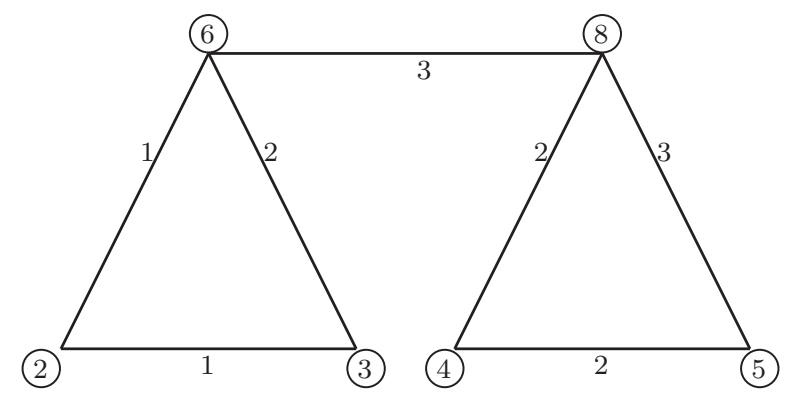

Figure 2. An Irregular assignment of $G=K_{2} \odot K_{2}$

For $n=2, G=K_{2} \odot K_{1,2}$. An irregular weighting of $G$ is given in figure 3 .

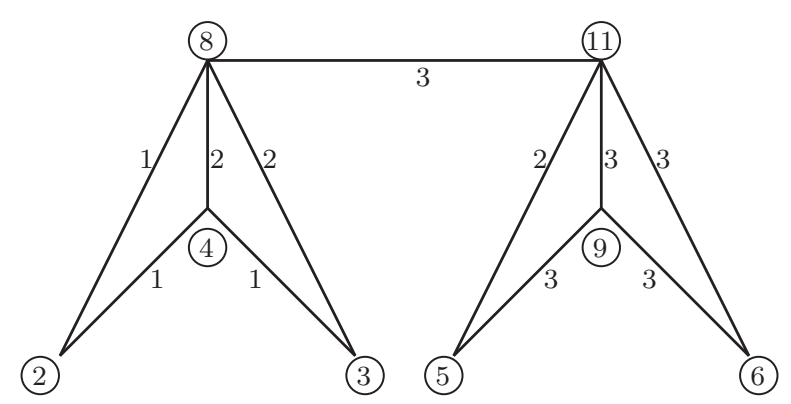

Figure 3. An Irregular assignment of $G=K_{2} \odot K_{1,2}$

\subsection{Case when $p>2$}

In this section, we consider the case when $p>2$. First we consider a special case which needs to be treated separately. Take $n=2$.

Theorem 2.7 Let $H$ be any graph having $p$ vertices such that $\delta(H) \geq 2$, then $s\left(H \odot K_{1,2}\right)=p+1$.

Proof. Let $x_{i}, y_{i}$ be the pendant vertices and $z_{i}$ be the center of $i^{\text {th }}$ star for $i=1,2,3, \cdots, p$. Let $u_{1}, u_{2}, u_{3}, \cdots, u_{p}$ be the vertices of $H$ and $d_{1} \leq d_{2} \leq \cdots \leq d_{p}$ be the degree sequence of $H$ such that $\operatorname{deg}\left(u_{i}\right)=d_{i}$. Assume that $i^{t h}$ copy of star is joined to the vertex $u_{i}$ of $H$.

$G=H \odot K_{1, n}$. Edges are $x_{i} z_{i}, y_{i} z_{i}, x_{i} u_{i}, y_{i} u_{i}, u_{i} z_{i}$ for $i=1,2, \cdots, p$ and edges of $H$. There will be $2 p$ vertices of degree 2 . Hence $s(G) \geq p+1$.

Define $f: E(G) \rightarrow Z^{+}$by $f\left(x_{i} z_{i}\right)=i, f\left(y_{i} z_{i}\right)=p, f\left(x_{i} u_{i}\right)=1, f\left(y_{i} u_{i}\right)=i+1, f\left(u_{i} z_{i}\right)=p+1$, and $f(e)=p+1$ for alle $\in$ $E(H)$

$w\left(x_{i}\right)=f\left(x_{i} z_{i}\right)+f\left(x_{i} u_{i}\right)=i+1$. These weights vary as $2,3, \cdots, p+1$.

$w\left(y_{i}\right)=f\left(y_{i} z_{i}\right)+f\left(y_{i} u_{i}\right)=p+i+1$. These weights vary as $p+2, p+3, \cdots, 2 p+1$.

$w\left(z_{i}\right)=f\left(x_{i} z_{i}\right)+f\left(y_{i} z_{i}\right)+f\left(z_{i} u_{i}\right)=i+p+p+1=2 p+i+1$. These weights vary as $2 p+2,2 p+3, \cdots, 3 p+1$.

$w\left(u_{i}\right)=f\left(x_{i} u_{i}\right)+f\left(y_{i} u_{i}\right)+f\left(z_{i} u_{i}\right)+d_{i}(p+1)=1+i+1+p+1+d_{i}(p+1)=p+3+d_{i}(p+1)+i$. Since $d_{1} \leq d_{2} \leq \cdots \leq d_{p}$, the weights of $u_{i}$ are distinct and are in increasing order. $\min w\left(u_{i}\right)=w\left(u_{1}\right)$. Also, since $d_{1} \geq 1, w\left(u_{1}\right)=p+3+d_{1}(p+1)+1 \geq$ $3 p+6>3 p+1$. Thus all these weights are distinct. So $s(G) \leq p+1$. Hence $s(G)=p+1$ if $\delta(H) \geq 2$.

Remark 2.8 The condition $\delta(H) \geq 2$ in theorem 2.8 is not necessary. There exist graphs $H$ with $\delta(H)=1$ and $s\left(H \odot K_{1,2}\right)=$ $p+1$ as shown in following example.

Example 2.9 Consider $H=P_{3}$ and $G=P_{3} \odot K_{1,2} . p=3$. The figure 4 gives an irregular weighting of $G$ so that $s(G)=4$. 


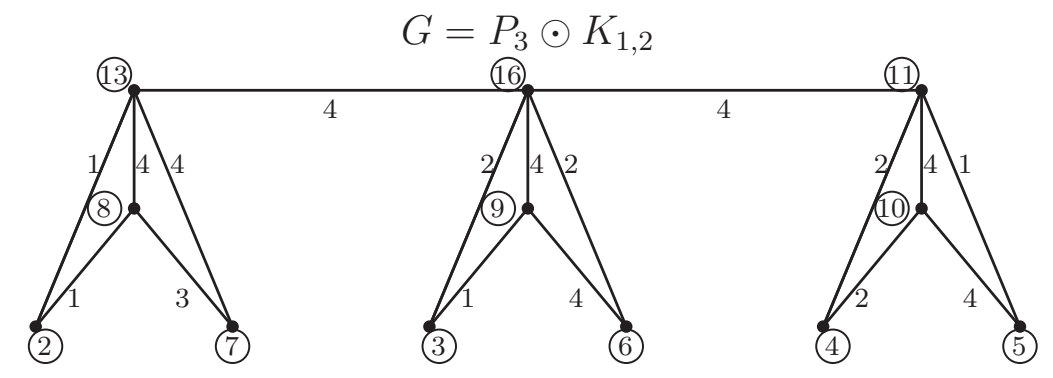

Figure 4. Irregular assignment of $G=P_{3} \odot K_{1,2}$

Now we consider the case when both $n, p \geq 3$. For the computation of weights, we use following two formulae.

Remark 2.10

$$
\begin{aligned}
T_{j} & =\sum_{i=1}^{n}\left\lceil\frac{j+(i-1) p}{2}\right\rceil \\
& =\frac{n(j+1)}{2}+\frac{n(n-1) p}{4} \quad \text { if p even, } j \text { odd } \\
& =\frac{n j}{2}+\frac{n(n-1) p}{4} \quad \text { if } p \text { even, } j \text { even } \\
& =\frac{n(2 j+1)}{4}+\frac{n(n-1) p}{4}+\frac{1}{4} \quad \text { if } n, p, j \text { odd } \\
& =\frac{n(2 j+1)}{4}+\frac{n(n-1) p}{4}-\frac{1}{4} \quad \text { if } n, p \text { odd, } j \text { even } \\
& =\frac{n(2 j+1)}{4}+\frac{n(n-1) p}{4} \quad \text { if } n \text { even, } p \text { odd }
\end{aligned}
$$

$$
\begin{aligned}
M_{j} & =\sum_{i=1}^{n}\left\lceil\frac{j+1+(i-1) p}{2}\right] \\
& =\frac{n(j+1)}{2}+\frac{n(n-1) p}{4} \quad \text { if } p \text { even, } j \text { odd } \\
& =\frac{n(j+2)}{2}+\frac{n(n-1) p}{4} \quad \text { if } p \text { even, } j \text { even } \\
& =\frac{n(2 j+3)}{4}+\frac{n(n-1) p}{4}-\frac{1}{4} \quad \text { if } n, p, j \text { odd } \\
& =\frac{n(2 j+3)}{4}+\frac{n(n-1) p}{4}+\frac{1}{4} \quad \text { if } n, p \text { odd }, j \text { even } \\
& =\frac{n(2 j+3)}{4}+\frac{n(n-1) p}{4} \quad \text { if } n \text { even, } p \text { odd }
\end{aligned}
$$

Theorem 2.11 Let $H$ be any graph without isolated vertices and having $p \geq 3$ vertices, then $s\left(H \odot K_{1, n}\right)=\left\lceil\frac{n p+1}{2}\right\rceil$ for $n \geq 3$.

Proof. Let $H$ be a graph without isolated vertices and having $p \geq 3$ vertices. Let $d_{1} \leq d_{2} \leq \cdots \leq d_{p}$ be the degree sequence of $H$. Let $u_{1}, u_{2}, u_{3}, \cdots, u_{p}$ be the vertices of $H$ with $\operatorname{deg}\left(u_{i}\right)=d_{i}$. Let $F_{1}, F_{2}, F_{3}, \cdots, F_{p}$ be the copies of star $K_{1, n}$. Assume $F_{j}$ is joined to the vertex $u_{j}$ for $j=1,2,3, \cdots, p$. Take the pendant vertices of $F_{j}$ as $u_{j i}$ for $i=1,2,3, \cdots, n$ and $w_{j}$ be center of the $\operatorname{star} F_{j}$.

$G=H \odot K_{1, n}$. Edges of $G$ are $w_{j} u_{j i}, u_{j} u_{j i}, u_{j} w_{j}$ for $j=1,2,3, \cdots, p$ and $i=1,2,3, \cdots, n$ and edges of $H$. $G$ has $n p$ vertices of degree 2 , so that $s(G) \geq\left\lceil\frac{n p+1}{2}\right\rceil$. Take $k=\left\lceil\frac{n p+1}{2}\right\rceil$.

Define $f: E(G) \rightarrow Z^{+}$by

$$
\begin{aligned}
f\left(w_{j} u_{j i}\right) & =\left\lceil\frac{j+(i-1) p}{2}\right\rceil \\
f\left(u_{j} u_{j i}\right) & =\left\lceil\frac{j+1+(i-1) p}{2}\right\rceil \\
f\left(u_{j} w_{j}\right) & =k \quad \text { if } p \text { is odd } \\
& =k-1 \quad \text { if } p \text { is even, jeven } \\
& =k \text { pis even, } j \text { odd } \\
f(e) & =k \text { for all } e \in E(H)
\end{aligned}
$$

Using this assignment the weights of vertices are calculated as follows.

$w\left(u_{j i}\right)=f\left(w_{j} u_{j i}\right)+f\left(u_{j} u_{j i}\right)=j+(i-1) p+1$. These weights vary as $2,3,4, \cdots, n p+1$.

$$
w\left(w_{j}\right)=\sum_{i=1}^{n}\left\lceil\frac{j+(i-1) p}{2}\right\rceil+f\left(w_{j} u_{j}\right)
$$




$$
w\left(u_{j}\right)=\sum_{i=1}^{n}\left\lceil\frac{j+1+(i-1) p}{2}\right\rceil+f\left(u_{j} w_{j}\right)+d_{j} k
$$

Case 1: $\quad p$ is even

If $T_{j}=\sum_{i=1}^{n}\left\lceil\frac{j+(i-1) p}{2}\right\rceil$, then

$$
T_{j}=\left\{\begin{array}{l}
\frac{n(j+1)}{2}+\frac{n(n-1) p}{4} \text { if } j \text { odd } \\
\frac{n j}{2}+\frac{n(n-1) p}{4} \text { if jeven }
\end{array}\right.
$$

Here $T_{j}$ will be same as $T_{j+1}$ for odd $j$.

$$
w\left(w_{j}\right)=\left\{\begin{array}{l}
\frac{n(j+1)}{2}+\frac{n(n-1) p}{4}+k \quad \text { if } \quad j \text { odd } \\
\frac{n j}{2}+\frac{n(n-1) p}{4}+k-1 \quad \text { if } j \text { even }
\end{array}\right.
$$

These weights are distinct and $\min w\left(w_{j}\right)=w\left(w_{2}\right)$. As $p$ is even $k=\frac{n p+2}{2} . w\left(w_{2}\right)=\frac{n(n+1) p}{4}+n$. As $n \geq 3, \frac{n(n+1) p}{4} \geq n p$. Then $w\left(w_{2}\right)=n+\frac{n(n+1) p}{4} \geq n+n p>n p+1=\max w\left(u_{j i}\right)$.

If $M_{j}=\sum_{i=1}^{n}\left\lceil\frac{j+1+(i-1) p}{2}\right\rceil$, then

$$
M_{j}=\left\{\begin{array}{lll}
\frac{n(j+1)}{2}+\frac{n(n-1) p}{4} & \text { if } & j \text { odd } \\
\frac{n(j+2)}{2}+\frac{n(n-1) p}{4} & \text { if } & j \text { even }
\end{array}\right.
$$

Here $M_{j}$ will be same as $M_{j+1}$ for even $j$.

$$
w\left(u_{j}\right)=\left\{\begin{array}{l}
\frac{n(j+1)}{2}+\frac{n(n-1) p}{4}+k+d_{j} k \quad \text { if } \quad j \text { odd } \\
\frac{n(j+2)}{2}+\frac{n(n-1) p}{4}+k-1+d_{j} k \quad \text { if } \quad j \text { even }
\end{array}\right.
$$

Since $d_{1} \leq d_{2} \leq d_{3} \leq \cdots \leq d_{p}$, the weights of $u_{j}$ are distinct and are in increasing order. $\min w\left(u_{j}\right)=w\left(u_{1}\right)$. Also $\max w\left(w_{j}\right)=w\left(w_{p-1}\right)$ as $p$ is even.

$$
\begin{aligned}
w\left(w_{p-1}\right) & =\frac{n(n-1) p}{4}+\frac{n p}{2}+\frac{n p}{2}+1 \\
& =\frac{n(n-1) p}{4}+n p+1
\end{aligned}
$$

Also $\min w\left(u_{j}\right)=w\left(u_{1}\right) \geq w\left(w_{p-1}\right)+n+1>w\left(w_{p-1}\right)=\max w\left(w_{j}\right)$. All weights are distinct. Hence $s(G) \leq k$. Thus $s(G)=k$.

Case 2: $\quad n$ is even and $p$ is odd. $k=\frac{n p}{2}+1$

$T_{j}=\frac{n(n-1) p}{4}+\frac{n(2 j+1)}{4}$. All these are distinct.

$w\left(w_{j}\right)=\frac{n(n-1) p}{4}+\frac{n(2 j+1)}{4}+\frac{n p}{2}+1$.

$\min w\left(w_{j}\right)=w\left(w_{1}\right)=\frac{n}{4}(n p+p+3)+1>n p+1$, as $n \geq 3$ even, $n \geq 4$. Thus $w\left(w_{1}\right)>\max w\left(u_{j i}\right)$.

$M_{j}=\frac{n(n-1) p}{4}+\frac{n(2 j+3)}{4}$. All these are distinct and are in increasing order.

$w\left(u_{j}\right)=\frac{n(n-1) p}{4}+\frac{n(2 j+3)}{4}+\frac{n p}{2}+1+d_{j}\left(\frac{n p}{2}+1\right)$.

Since $d_{1} \leq d_{2} \leq \cdots \leq d_{p}$, the weights of $u_{j}$ are distinct and are in increasing order.

Also $\max w\left(w_{j}\right)=w\left(w_{p}\right)=\frac{n}{4}(n p+p+1)+\frac{n p}{2}+1$ and $\min w\left(u_{j}\right)=\frac{n(n-1) p}{4}+\frac{5 n}{4}+\frac{n p}{2}+1+d_{1}\left(\frac{n p}{2}+1\right) \geq w\left(w_{p}\right)+n+1>w\left(w_{p}\right)$. Hence all these weights are distinct. Thus $s(G)=k$.

Case 3: $\quad$ Both $n$ and $p$ are odd. $k=\frac{n p+1}{2}$.

$$
T_{j}= \begin{cases}\frac{n(n-1) p}{4}+\frac{n(2 j+1)}{4}+\frac{1}{4} & \text { if } j \text { odd } \\ \frac{n(n-1) p}{4}+\frac{n(2 j+1)}{4}-\frac{1}{4} & \text { if } j \text { even }\end{cases}
$$


$w\left(w_{j}\right)=T_{j}+\frac{n p+1}{2}$. All these are distinct and are in increasing order. $\min w\left(w_{j}\right)=w\left(w_{1}\right) \geq n p+3$ as $n \geq 3$. Hence $\min w\left(w_{j}\right)>n p+1$.

$$
\begin{aligned}
w\left(u_{j}\right) & =M_{j}+\frac{n p+1}{2}+d_{1}\left(\frac{n p+1}{2}\right) \\
& = \begin{cases}\frac{n(n-1) p}{4}+\frac{n(2 j+3)}{4}-\frac{1}{4}+d_{1}\left(\frac{n p+1}{2}\right)+k+\frac{n p+1}{2} & \text { if } j \text { odd } \\
\frac{n(n-1) p}{4}+\frac{n(2 j+1)}{4}+\frac{1}{4}+d_{1}\left(\frac{n p+1}{2}\right)+k+\frac{n p+1}{2} & \text { if } j \text { even }\end{cases}
\end{aligned}
$$

As $n>2$ and $d_{1} \leq d_{2} \leq \cdots \leq d_{p}$, all these weights $w\left(u_{j}\right)$ are distinct and are in increasing order. $\max w\left(w_{j}\right)=w\left(w_{p}\right)=$ $\frac{n(n-1) p}{4}+\frac{n(2 p+1)}{4}+\frac{1}{4}+\frac{n p+1}{2} . \min w\left(u_{j}\right)=\frac{n(n-1) p}{4}+\frac{5 n}{4}-\frac{1}{4}+\frac{n p+1}{2}+d_{1}\left(\frac{n p+1}{2}\right)>w\left(w_{p}\right)$ as $d \geq 1$. All these weights are distinct. Thus we have $s(G)=k$.

Remark 2.12 The condition $\delta(H)>0$ in theorem 2.11 is not necessary. There exist graphs $H$ with $\delta(H)=0$ and $s\left(H \odot K_{1, n}\right)=\left\lceil\frac{n p+1}{2}\right\rceil$.

Example 2.13 Consider $H=K_{1} \cup K_{2}$ and $G=\left(K_{1} \cup K_{2}\right) \odot K_{1,3} \cdot n=3, p=3,\left\lceil\frac{n p+1}{2}\right\rceil=5$. The figure 5 gives an irregular weighting of $G$ so that $s(G)=5$.

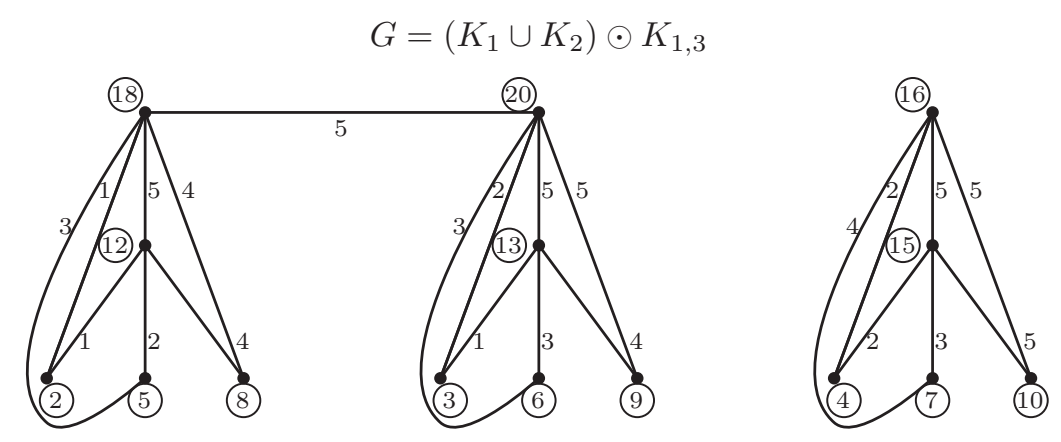

Figure 5. Irregular assignment of $G=\left(K_{1} \cup K_{2}\right) \odot K_{1,3}$

\section{References}

Chartrand, G., Jacobson, M. S., Lehel, J., Ollerman, O. R., Ruiz, S., \& Saba. (1988). Irregular networks. Congr. Numer, 64, 197-210.

Ebert, G., Hemmeter, J., Lazebmik, F., \& Wolder, A. J. (1990). On irregularity strength of some Graphs. Congr. Numer, $71,39-52$.

Faudree, R. J., Jacobson, M. S., Kinch, L., \& Lehel, J. (1991). Irregularity strength of Dense Graphs. Discrete Mathematics, 91, 45-59. http://dx.doi.org/10.1016/0012-365X(91)90161-T

Jacobson, M., \& Lehel, J. (1998). A bound for the strength of an irregular network, manuscript.

Jinnah, M. I., Santhosh Kumar, K. R. (2012). Irregularity strength of corona product of two graphs, Proceedings of National Seminar on Algebra, Analysis and Discrete Mathematics, University of Kerala, Thiruvananthapuram,Kerala, India Editors: A R Rajan, C Jayasri and L John, pp 191-199.

Joseph, A. G. (2010). A Dynamic survey of Graph Labeling. The Electronic Journal of Combinatorics, 17,\#DS 6.

\section{Copyrights}

Copyright for this article is retained by the author(s), with first publication rights granted to the journal.

This is an open-access article distributed under the terms and conditions of the Creative Commons Attribution license (http://creativecommons.org/licenses/by/3.0/). 